\title{
A NEW DIRECT READING VACUUM GAGE.
}

BY W. H. FARR,

Central Scientific Company, Chicago, Ill.

When the Central Seientific Company began producing the Cenco-Nelson Vacuum Pump in quantities one problem which presented itself to the laboratory was that of a satisfactory routine test of the vacuum produced by this pump. The chief requirements of such a test are, that it must be quick, reliable, and "foolproof." The McLeod gage was used for some time, but there are some objections to its use as a routine test in quantity production. In the first place it is rather slow. It not only takes a little time to fill the bulb and empty it again but besides it is necessary to take a series of readings over a period of time to see whether or not the vacuum is improving. Furthermore the apparatus is rather delicate for routine use, and can be easily ruined by careless handling. If oil is accidentally allowed to get into the bulb, the cleaning and drying of the glassware involves considerable loss of time. In case the glassware is broken or if mercury is spilled, renewal or recalibration is rather expensive. In view of these facts, the laboratory began to investigate other means of measuring a vacuum, which would be more suitable for our conditions.

The variety of effects displayed by electrical discharges in rarified gases suggested the idea of using some of these phenomena to indicate the degree of vacuum produced by a pump. The problem was to determine which of the characteristics of the vacuum discharge could be measured quantitatively with sufficient accuracy to serve as a measure of the vacuum.

Of the two sizes of the Cenco-Nelson Pump (2 stage and 3 stage) the 2 stage is guaranteed to produce a vacuum of $.10 \mathrm{~mm}$. and the 3 stage of $.05 \mathrm{~mm}$. Occasionally a 3 stage pump will be found which will go as low as .02 $\mathrm{mm}$.

Before a vacuum of $.10 \mathrm{~mm}$. has been reached the Faraday's dark space has become well defined, and occupies a considerable portion of the tube. The positive column is broken up into well defined striations, which become larger and fewer as the vacuum improves. It was first attempted to use the length and form of the positive column as the quantity to be measured. This gives a fair indication of the vacuum, but is not sufficiently reproducible to serve as a reliable test.

It is affected by such factors as the size and proportions of the tube, the form of the electrodes, the dryness of the tube and the 
frequency of the current. This unreliability is especially noticeable in the neighborhood of $.05 \mathrm{~mm}$. and above, which is just where the greatest accuracy is desired.

Another phenomenon which was investigated was the appearance of the apple green fluoresence which indicates the beginning of the production of cathode rays. The difficulty here was that there is nothing about this color which can be measured quantitatively, and its appearance is affected by some of the conditions of the test, such as the condition of the tube, the shape of electrodes and the strength of the spark coil.

An attempt was made to test the vacuum in the tube by measuring the current flowing through the tube with a hot wire milliammeter, but this method was abandoned. It was also attempted to use a spark gap in parallel with the tube, the length of the gap to be adjusted so that it would spark over at the desired vacuum. The resistance of the tube proved to be too low for this method to be a success.

It was finally found that the most satisfactory characteristic of the vacuum discharge to be measured quantitatively is the Crooker dark space. This dark space begins to appear around the negative electrode at a vacuum of about one millimeter and increases up to a vacuum of about .02 or .01 when it becomes too hazy and indefinite to measure. The dark space is most easily measured in a tube having disk shaped electrodes. In such a tube it appears as a cylindrical shaped space with a well de-

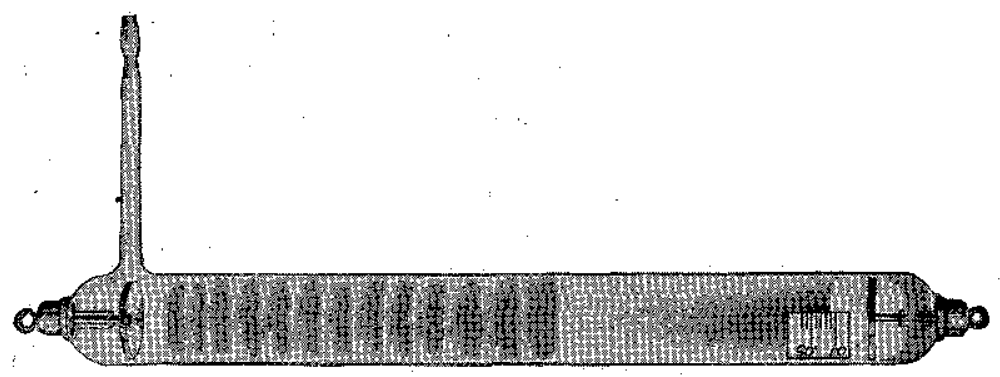

fined edge, as shown in Fig. 1, whose length can be measured with an accuracy of about 1-2 $\mathrm{mm}$. It will be noted that the range over which this dark space is measurable covers the range of the vacuum produced by the Cenco-Nelson Pump.

To determine the reliability of the measurements of this dark space as an index to the degree of vacuum, it was necessary to determine how much it is affected by the conditions of the test. 
such as the individual characteristics of the tube, voltage of the battery, frequency of the vibrator, voltage of the coil, etc. It was found that the length of the dark space is not affected by the diameter of the tube but there is a certain relation between the diameter of the electrodes and the diameter of the tube, and also between the length of the tube and its diameter, which gives the most clear and definite reading.

To determine if the dark space varies from one tube to another depending on the individual characteristics of the tube, a number of tubes were made up, as nearly.indentical as possible in form and dimensions. These were calibrated by the McLeod gage and the measurements compared.' It was found that in tubes of the same form and dimensions the length of the dark space is an accurately reproducible quantity. The effect of the voltage of the coil and the frequency of the vibrator were also investigated. The only effects of changes in the voltage of the coil is to vary the intensity of the discharge, it having no effect on the lingth of the cathode dark space. The same is true of the frequency of the vibrator.

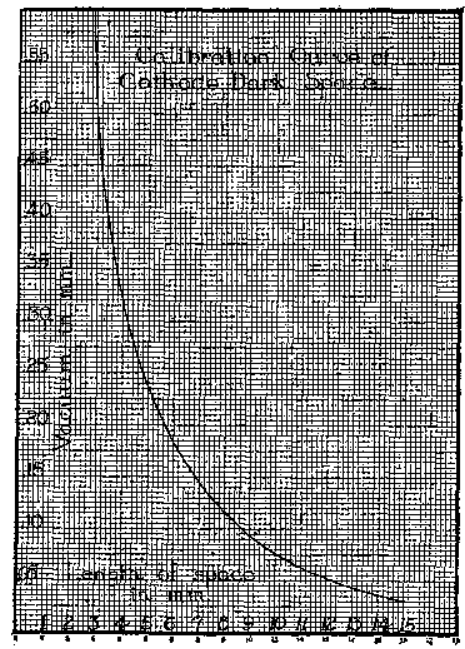

The relation between the length of the dark space and the vacuum is shown by the curve in Fig. 2 . It will be noted that the rate of change in length becomes more rapid as the vacuum becomes higher. This is a decided advantage, as it makes the accuracy of the readings increase as the vacuum increases. In the tests of the Cenco-Nelson Pumps the readings come near 
the lower end of the curve, at which point it is easily possible to graduate the scale on the tube to read $.01 \mathrm{~mm}$. directly, which is a satisfactory degree of accuracy for this test.

During the development work on this test, all the work was done in the dark room. When it was perfected, however, a shade was constructed which made it possible to use the equipment in daylight. This was in the form of a wooden box whose dimensions were slightly larger than those of the tube, and which was painted black inside and outside. The tube is observed through a wide slot in the front side. The tube is held in spring clips, so that it is easily removable, and the connections are made through binding posts on the outside.

In conclusion, the specific advantages which are claimed for this test are the following:

1. As the connections are shorter and there is a smaller volume of air to exhaust, the desired vacuum is reached more quickly than when using a MeLeod gage.

2. The reading is instantaneous, whereas some little time is required to read a McLeod gage.

3 . This test gives a continuous indication of the vacuum, while with the McLeod gage it is necessary to take a series of readings to determine whether the vacuum is improving or not.

4. As the scale on the tube is direct reading, it requires no experience or practice to interpret the result, nor is there any source of error due to a zero setting.

5. The accuracy of this method increases with the degree of vacuum.

6. This test does not require the handling of mercury or expensive glassware, making it more suitable for a routine test.

7. The complete apparatus is light and portable.

8. In case of breakage of the glassware, renewal is not expensive.

9. A number of testing sets can be operated from the same coil and battery, it being only necessary to duplicate the vacuum tube.

10. The accuracy of the test being independent of the voltage or frequency, there are no meter readings or corrections. 